\title{
Development of a New Cracked Mindlin Plate Element
}

\author{
Chengyin Liu, ${ }^{1,2}$ John DeWolf, ${ }^{2}$ and Jeong-Ho Kim ${ }^{2}$ \\ ${ }^{1}$ Center of Bridge and Structural Engineering, Harbin Institute of Technology Shenzhen Graduate School, Shenzhen 518055, China \\ ${ }^{2}$ Department of Civil and Environmental Engineering, University of Connecticut, 261 Glenbrook Road, Unit 2037, Storrs, \\ CT 06269, USA
}

Correspondence should be addressed to Chengyin Liu, chengyin.liu08@gmail.com

Received 18 May 2011; Accepted 15 June 2011

Academic Editors: Y. Goto and I. Raftoyiannis

Copyright (C) 2011 Chengyin Liu et al. This is an open access article distributed under the Creative Commons Attribution License, which permits unrestricted use, distribution, and reproduction in any medium, provided the original work is properly cited.

This work addresses the development of a new four-noded rectangular Mindlin plate bending element (MP4C) with a crack which consists of three degrees of freedom (DOF) at each corner node. The crack in the element is assumed to be not closed and nonpropagating. The crack affects the elastic strain energy and the flexibility matrix of the element, whereas the mass matrix remains unchanged. The complete element stiffness matrix is constructed as the inverse of the combined flexibility matrix of both noncracked and cracked elements. To evaluate the behavior of the proposed cracked Mindlin plate element, numerical examples are provided. They are based on developing user subroutines in ABAQUS. The finite element analysis results using the developed plate element are in excellent agreement with those reported in previous work. The cracked plate element developed in this paper provides a simple and robust approach to model the real service conditions in plate-like structures.

\section{Introduction}

Civil structures such as highway bridges are subject to a complex environment during their lifetime. In the past two decades, researchers at the University of Connecticut have been exploring different approaches to conduct longterm monitoring for application to bridges. These efforts include vibration monitoring on different types of concrete bridges, nondestructive load testing on steel girder bridges, environmental evaluation of a multispan continuous bridges, and the development of a variety of damage detection methods based on both experimental and analytical bridge models $[1,2]$. This paper reports on the study of novel techniques that can be used for finite element analysis of cracked plate-like structures to consider their real service conditions.

A crack or local defect is known to affect the dynamic response of a structural member. The first attempts to quantify defects were made by Thomson [3] and Kirmsher [4]. In their investigations, the effect of a notch on the structure flexibility was simulated by a local bending moment or reduced section, with magnitudes measured by experiments. Since 1950s, as researchers started to realize that the local flexibility will reduce the stiffness of a structural member and subsequently reduce its natural frequency, the analysis of the local flexibility of a cracked region of structural element was largely considered for the structural damage identification.

In the following a few decades, the concept of local flexibility in crack regions has been extensively applied in developing techniques of finite elements with cracks. In those elements, the crack was represented by a localized compliance, and the stiffness matrix of the cracked element was expressed as a function of the crack depth and location. While the local flexibility approach has been developed for elements like truss, beam, and rod, few researches have dealt with plate elements with cracks. Guan-Liang et al. [5] discussed an approximate formulation of a cracked plate bending element by finding the strain energy under the occurrence of crack. They derived the element stiffness matrix from the integration of stress intensity factors (SIFs). Almost at the same time, Krawczuk [6] independently developed a similar cracked plate finite element with in-plane forces, and later extended his method to develop shell element with a through crack and plate element with an elastoplastic through crack $[7,8]$. These existing plate elements, however, neglect the effects of transverse shear, and so 


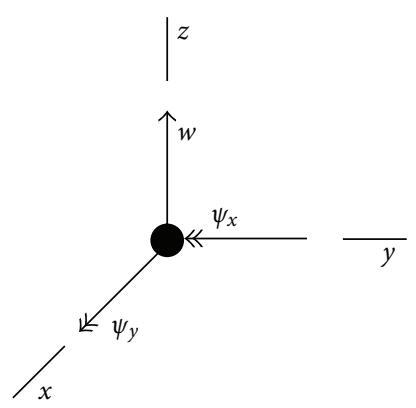

FIgure 1: Typical nodal DOF of a corner node in the Mindlin plate element.

they are insufficient for practical engineering applications when it is necessary to model plate bending behaviors. In engineering applications, many plate members must be modeled as moderately thick plate elements due to the relatively large thickness in comparison with other two dimensions.

Mindlin [9] presented a complete theory for the shear deformable plates in static and dynamics, referred to as the first-order shear deformation theory. This theory, which accounts for transverse shear deformations, is better suited for the solution of plate bending problems than the Kirchhoff plate theory. A wealth of further analytic, numerical, and experimental investigations done by other investigators with the Mindlin theory make it feasible for use as a rigorous cracked plate bending element. Thus this paper develops a new rectangular cracked Mindlin plate bending finite element using the local flexibility approach and explicitly considering three independent fracture modes [10]. This cracked element possesses four corner nodes with three engineering degrees of freedom (DOF) at each node. In the derivation of the stiffness matrix, selective numerical integration is used to overcome the shear-locking problem. This paper also explores a practical engineering analysis tool that can readily model the dynamic response of structures in the presence of discrete cracks, with reasonable engineering accuracy. This paper provides a framework for incorporating the developed elements into the commercial finite element software ABAQUS [11] through a user element subroutine. The behaviors of the developed noncracked and cracked elements are examined and compared with results presented by other researchers and elements available in ABAQUS. The examples presented in this paper address the performance of the proposed elements for use in problems with either thin or thick plates.

\section{Fundamental Relations in the Mindlin Plate Theory}

Kirchhoff elements were introduced earlier by the influence of the classical plate theory. Mindlin elements appeared later with the assumption that a straight line originally normal to the plate midsurface remains straight but not normal after deformation. A shear factor was introduced to take account of the fact that the shear strain through the plate thickness is not uniformly distributed. Figure 1 shows the typical nodal DOFs in the Mindlin plate element. The inclusion of transverse shear effects in the classical plate theory indicates that the cross-sectional rotations $\psi_{x}$ and $\psi_{y}$ are no longer expressed solely in terms of the deflection $w$. Thus, there exist three basic reference quantities, namely, $w, \psi_{x}$ and $\psi_{y}$, at the midsurface.

2.1. Displacement Fields. Considering the DOF in the Mindlin plate element as shown in Figure 1, the displacement fields in the Cartesian coordinate system are given as [12]

$$
\begin{aligned}
& u=u(x, y, z)=-z \psi_{x}(x, y), \\
& v=v(x, y, z)=-z \psi_{y}(x, y), \\
& w=w(x, y, z)=w(x, y),
\end{aligned}
$$

where $w, \psi_{x}$, and $\psi_{y}$ are the lateral ( $z$-direction) deflection and the two normal rotations of the midsurface, respectively.

2.2. Strain-Curvature Relations. For small displacements and rotations, strains are given by [12]:

$$
\begin{gathered}
\mathcal{E}_{x}=-z \psi_{x, x}, \quad \varepsilon_{y}=-z \psi_{y, y}, \quad \gamma_{x y}=-z\left(\psi_{x, y}+\psi_{y, x}\right), \\
\gamma_{y z}=w_{, y}-\psi_{y}, \quad \gamma_{z x}=w_{, x}-\psi_{x},
\end{gathered}
$$

where a comma denotes differentiation with respect to the following subscript. Equation (2) is the basis of the Mindlin plate theory, which allows nonzero transverse shear strains. The bending curvatures are given by

$$
\kappa_{x}=\psi_{x, x}, \quad \kappa_{y}=\psi_{y, y}, \quad \kappa_{x y}=\left(\psi_{x, y}+\psi_{y, x}\right) .
$$

Both curvatures and transverse shear strains appeared in the vector $\{\kappa\}$ as follows:

$$
\{\kappa\}=\left\{\kappa_{x}, \kappa_{y}, \kappa_{x y},-\gamma_{z x},-\gamma_{y z}\right\}^{T}
$$

2.3. Constitutive Relations. For homogeneous, isotropic, and linearly elastic material, the moment-curvature relations for the Mindlin plate are

$$
\left\{M_{c}\right\}=[D]\{\kappa\},
$$

in which $\left\{M_{c}\right\}$ is the moment vector including $\left\{M_{x}\right.$, $\left.M_{y}, M_{x y}, Q_{x}, Q_{y}\right\}^{T}$ and $[D]$ is the rigidity matrix, analogous to flexural stiffness EI of a beam. The forces and associated stresses on the plate are described in later sections. 


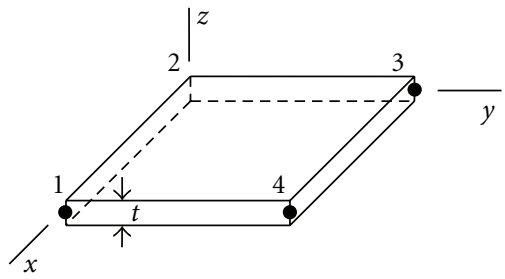

Figure 2: A Mindlin plate finite element (MP4) with four corner nodes.

\section{Mindlin Plate Element (MP4)}

Figure 2 shows a four-node Mindlin plate element with 3 DOF at each node. The lateral displacement and rotation fields of the MP4 element with $n$ nodes can be given by

$$
\begin{gathered}
\left\{\begin{array}{c}
w \\
\psi_{x} \\
\psi_{y}
\end{array}\right\}=\sum_{i=1}^{n}\left(\begin{array}{ccc}
N_{i} & 0 & 0 \\
0 & N_{i} & 0 \\
0 & 0 & N_{i}
\end{array}\right)\left\{\begin{array}{l}
w_{i} \\
\psi_{x_{i}} \\
\psi_{y_{i}}
\end{array}\right\} \quad \text { or } \\
\{u\}=[N]\{d\},
\end{gathered}
$$

where the same shape functions are used for each displacement component.

With $\{u\}=\left[w, \psi_{x}, \psi_{y}\right]^{T}$, we obtain from (2) and (4)

$$
\begin{gathered}
\{k\}=\left\{\begin{array}{c}
\psi_{x, x} \\
\psi_{y, y} \\
\psi_{x, y}+\psi_{y, x} \\
\psi_{x}-w_{, x} \\
\psi_{y}-w_{, y}
\end{array}\right\}=[\partial]\{u\}, \\
\text { where }[\partial]=\left[\begin{array}{ccc}
0 & \frac{\partial}{\partial x} & 0 \\
0 & 0 & \frac{\partial}{\partial y} \\
0 & \frac{\partial}{\partial y} & \frac{\partial}{\partial x} \\
\frac{-\partial}{\partial x} & 1 & 0 \\
\frac{-\partial}{\partial y} & 0 & 1
\end{array}\right]
\end{gathered}
$$

From (6) and (7), we obtain for an element with 4 nodes

$$
\{k\}=[B]_{5 \times 3 n}\{d\},
$$

where $[B]=[\partial][N]=\left[\begin{array}{ccccc}0 & N_{1, x} & 0 & \cdots & 0 \\ 0 & 0 & N_{1, y} & \cdots & N_{n, y} \\ 0 & N_{1, y} & N_{1, x} & \cdots & N_{n, x} \\ -N_{1, x} & N_{1} & 0 & \cdots & 0 \\ -N_{1, y} & 0 & N_{1} & \cdots & 0\end{array}\right]$.

The element stiffness matrix $[K]$ is

$$
[K]=\int[B]^{T}[D][B] d A .
$$

\section{Cracked Mindlin Plate Element (MP4C)}

Figure 3(a) illustrates a quadrilateral plate with a through crack of $2 a$ and subjected to twelve element forces $S_{n}(n=$ 1-12). These twelve forces are related by three equations of equilibrium, and consequently only nine of these forces are linearly independent. Figure 3(b) shows the independent force system (Przemieniecki [13]). The matrix [T], given in Appendix A.1, was used to transform the nodal forces $S_{1}-S_{12}$ to the independent ones $F_{1}-F_{9}$. The stiffness matrix $\left[K_{c}\right]$ of the MP4C element can be now written as

$$
\left[K_{c}\right]=[T]^{T}\left[C_{f}\right]^{-1}[T]
$$

where $\left[C_{f}\right]^{-1}$ denotes the inverse matrix of $\left[C_{f}\right]=$ $\left[C_{f}\right]^{0+}\left[C_{f}\right]^{1}$ in which $\left[C_{f}\right]^{0}$ is the flexibility matrix of the noncracked element and $\left[C_{f}\right]^{1}$ is the flexibility matrix due to the presence of the crack. The details of the derivation of these two flexibility matrices are given in the next two sections.

4.1. Flexibility Matrix of the Noncracked Element. The elemental flexibility matrix is given by Przemieniecki [13]

$$
f=\int_{V}[e]^{T}[\Phi][e] d V
$$

in which matrix $[e]$ represents the approximate stress distribution due to unit force and $[\Phi]$ is the inverse matrix of flexural rigidity. Figure 4(a) depicts internal stresses on the plate cross-section. These stresses are distributed over the thickness of the plate and they produce bending moments, twisting moments, and vertical shear forces per unit of length in the $x y$ plane, as shown in Figure 4(b). The inplane stresses $\sigma_{x}, \sigma_{y}$, and $\tau_{x y}$, vary linearly with $z$, whereas transverse shear stresses $\tau_{y z}$ and $\tau_{z x}$ usually vary parabolically through the thickness. The relationships between these stresses with the moments and forces are given by

$$
\begin{gathered}
\sigma_{x x}=\frac{12 z}{t^{3}} M_{x}, \quad \sigma_{y y}=\frac{12 z}{t^{3}} M_{y}, \quad \tau_{x y}=\frac{12 z}{t^{3}} M_{x y}, \\
\tau_{y z}=\frac{3}{2 t}\left[1-\left(\frac{2 z}{t}\right)^{2}\right] Q_{y}, \quad \tau_{z x}=\frac{3}{2 t}\left[1-\left(\frac{2 z}{t}\right)^{2}\right] Q_{x} .
\end{gathered}
$$

Using (12) and assuming that the normal stresses vary linearly and the shearing stresses are constant along the plate edges, we can obtain the matrix $[e]$ as 


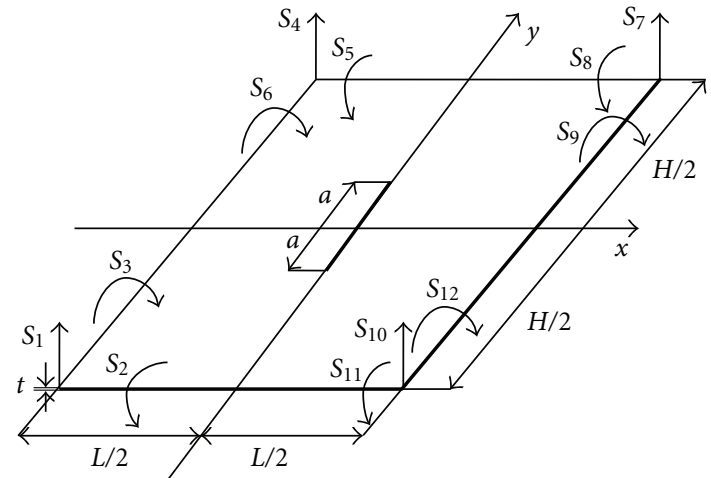

(a)

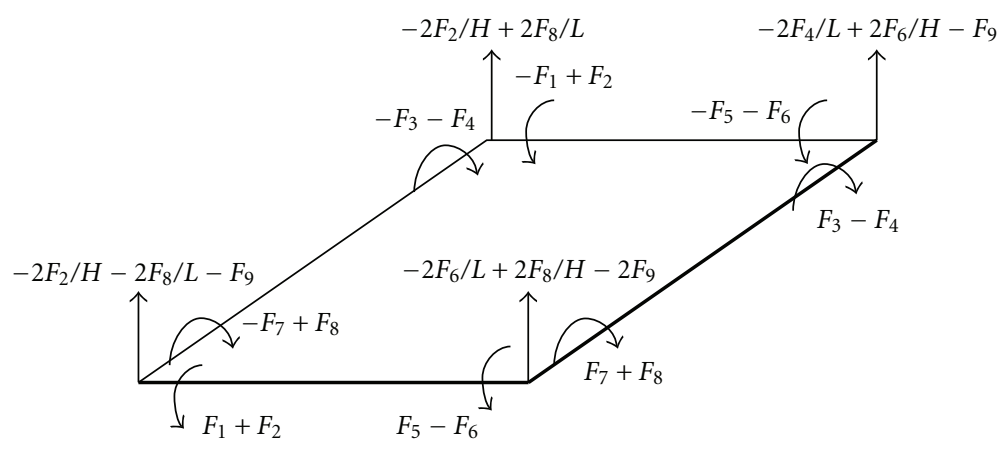

(b)

Figure 3: (a) A cracked Mindlin plate element (MP4C), (b) independent force system for MP4C element.

$$
[e]^{T}=\frac{12 z}{t^{3}}\left[\begin{array}{ccccc}
0 & \frac{2}{L}(2-3 \xi) & 0 & 0 & 0 \\
0 & \frac{2}{L}(2-3 \xi)(1-2 \eta) & 0 & 0 & 0 \\
\frac{2}{H}(-1+3 \eta) & 0 & 0 & 0 & 0 \\
\frac{2}{H}(-1+3 \eta)(1-2 \xi) & 0 & 0 & 0 & 0 \\
0 & \frac{2}{L}(-1+3 \xi) & 0 & 0 & 0 \\
0 & \frac{2}{L}(-1+3 \xi)(-1+2 \eta) & 0 & 0 & 0 \\
\frac{2}{L}(2-3 \eta) & 0 & 0 & 0 & 0 \\
\frac{2}{L}(2-3 \eta)(-1+2 \xi) & 0 & 0 & 0 \\
0 & 0 & 1 \frac{t^{2}}{8 z}\left[1-\left(\frac{2 z}{t}\right)^{2}\right] \frac{t^{2}}{8 z}\left[1-\left(\frac{2 z}{t}\right)^{2}\right.
\end{array}\right] .
$$

in which $\xi, \eta$ are the local coordinates for the element. Substituting (13) into (11), we can derive the flexibility matrix $\left[C_{f}\right]^{0}$ which is given in Appendix A.2.

4.2. Flexibility Matrix due to Crack. We have the following relationship due to crack in the element:

$$
c_{i, j}^{1}=\frac{\partial^{2} U^{1}}{\partial F_{i} \partial F_{j}},
$$

where $F_{i}$ denotes independent nodal forces and $U^{1}$ is the strain energy induced due to crack given in Ugural [14]

$$
U^{1}=\frac{1}{E} \int_{A}\left(\sum_{i=1}^{9} K_{I, i}^{2}+\sum_{i=1}^{9} K_{I I, i}^{2}+(1+v) \sum_{i=1}^{9} K_{I I I, i}^{2}\right) d A^{1},
$$

where $K_{j i}$ are stress intensity factors for two modes of crack evolution, and $A^{1}$ is the area of the crack. For a plate with uniform thickness $t$ containing a through crack of $2 a$, and subjected to the $F_{1}-F_{9}$ independent forces, the SIFs are given by Sih and Liebowitz [15]:

$$
\begin{aligned}
& K_{I}^{*}=\frac{1}{\sqrt{\pi a}} \int_{-a}^{a} \sigma_{x x}(0, y) \sqrt{\frac{a+y}{a-y}} d y, \\
& K_{I I}^{*}=\frac{-1}{\sqrt{\pi a}} \int_{-a}^{a} \tau_{x y}(0, y) \sqrt{\frac{a+y}{a-y}} d y, \\
& K_{I I}^{*}=\frac{1}{\sqrt{\pi a}} \int_{-a}^{a} \tau_{y z}(0, y) \sqrt{\frac{a+y}{a-y}} d y,
\end{aligned}
$$

where $\sigma_{x x}, \tau_{x y}$, and $\tau_{y z}$ are normal, in-plane shear, and transverse shear stress acting on the crack face given by

$$
\begin{aligned}
& \sigma_{x x}(0, y)=\frac{24 z}{H t^{3}}\left[\left(\frac{1}{2}+\frac{3 y}{H}\right) F_{3}+\left(\frac{1}{2}-\frac{3 y}{H}\right) F_{7}\right], \\
& \tau_{x y}(0, y)=\frac{12 z}{t^{3}} F_{9}, \\
& \tau_{y z}(0, y)=\frac{3}{2 t}\left[1-\left(\frac{2 z}{t}\right)^{2}\right] F_{9} .
\end{aligned}
$$




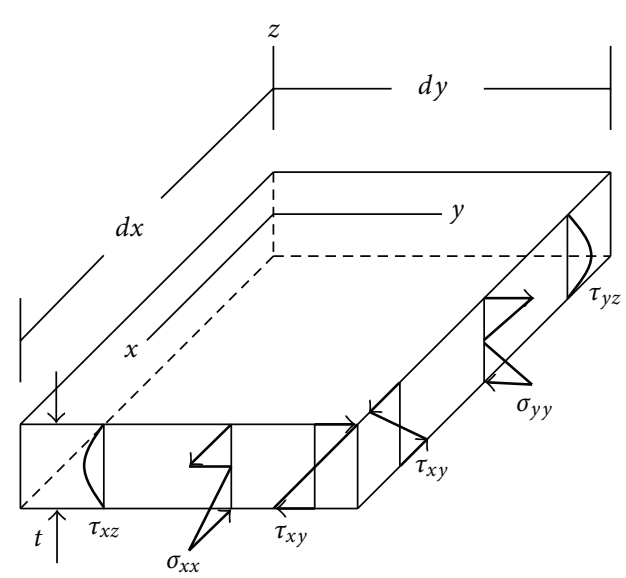

(a)

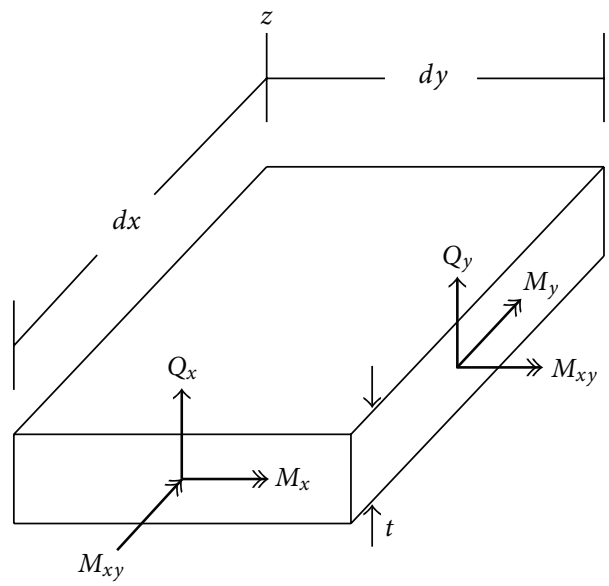

(b)

FIGURE 4: (a) Stress distribution on a differential element of a plate, (b) moments and transverse shear forces associated with internal stresses.

Substituting (17) into (16), the SIFs for an elastic nonpropagating crack are given by

$$
\begin{aligned}
K_{I}^{*} & =\frac{24 z}{H t^{3}}\left[F_{3}\left(\frac{1}{2}+\frac{3 a}{2 H}\right)+F_{7}\left(\frac{1}{2}-\frac{3 a}{2 H}\right)\right] \sqrt{\pi a}, \\
K_{I I}^{*} & =-\frac{12 z}{t^{3}} F_{9} \sqrt{\pi a}, \\
K_{I I I}^{*} & =\frac{3}{2 t}\left[1-\left(\frac{2 z}{t}\right)^{2}\right] F_{9} \sqrt{\pi a} .
\end{aligned}
$$

The above SIFs are suitable for an infinite plate only. To take into account finite dimensions of the element, a geometric correction factor can be used [16]

$$
\begin{aligned}
Y(g)= & 1.0+0.01876\left(\frac{2 a}{H}\right)+0.1825\left(\frac{2 a}{H}\right)^{2}+2.024\left(\frac{2 a}{H}\right)^{3} \\
& -2.4316\left(\frac{2 a}{H}\right)^{4} .
\end{aligned}
$$

Combining the geometric correction factor leads to

$$
\begin{aligned}
K_{I} & =\frac{24 z}{H t^{3}}\left[F_{3}\left(\frac{1}{2}+\frac{3 a}{2 H}\right)+F_{7}\left(\frac{1}{2}-\frac{3 a}{2 H}\right)\right] \sqrt{\pi a} Y(g), \\
K_{I I} & =-\frac{12 z}{t^{3}} F_{9} \sqrt{\pi a} Y(g), \\
K_{I I I} & =\frac{3}{2 t}\left[1-\left(\frac{2 z}{t}\right)^{2}\right] F_{9} \sqrt{\pi a} Y(g) .
\end{aligned}
$$

The expression for the mode III SIF $\left(K_{I I I}\right)$ in $(20)$ is first derived in this paper. We explicitly use the independent three fracture modes to evaluate the flexibility matrix of the cracked Mindlin plate element. For this approach, there is no need to incorporate fracture mode correction functions [58], used to transfer from the Kirchhoff theory to the Mindlin theory.
Substituting (15) and (20) into (14) leads to the following nonzero terms in the flexibility matrix of the MP4C element with crack:

$$
\begin{aligned}
& c_{33}^{1}=4\left[\int_{-g_{k}}^{g_{k}} g\left(\frac{1}{2}+\frac{3 g}{4}\right)^{2} Y^{2}(g) d g\right], \\
& c_{37}^{1}=2\left[\int_{-g_{k}}^{g_{k}} g\left(\frac{1}{2}+\frac{3 g}{4}\right)\left(\frac{1}{2}-\frac{3 g}{4}\right) Y^{2}(g) d g\right], \\
& c_{77}^{1}=4\left[\int_{-g_{k}}^{g_{k}} g\left(\frac{1}{2}-\frac{3 g}{4}\right)^{2} Y^{2}(g) d g\right], \\
& c_{99}^{1}=\left[1+(1+v) \frac{t^{2}}{10}\right] H^{2}\left[\int_{-g_{k}}^{g_{k}} g Y^{2}(g) d g\right] .
\end{aligned}
$$

The flexibility matrix $\left[C_{f}\right]^{1}$ derived in this procedure is given in Appendix A.3.

\section{Numerical Examples}

5.1. Example 1: Free Vibration of a Simply Supported Plate. In this example, the natural frequencies of free vibration of a simply supported square plate are calculated. The geometric data employed are the length of the edge of the plate $L=$ $0.1 \mathrm{~m}$, the elastic modulus $E=2.04 \times 10^{11} \mathrm{~N} / \mathrm{m}^{2}$, the Poisson ratio $v=0.3$, and the density $\rho_{0}=7860 \mathrm{~kg} / \mathrm{m}^{3}$. Three values of plate thickness, $t=0.001 \mathrm{~m}, t=0.005 \mathrm{~m}$, and $t=0.01 \mathrm{~m}$, are considered representing the thin $(t / L=1 \%)$, moderate $(t / L=5 \%)$, and thick plate $(t / L=10 \%)$, respectively.

The natural frequencies in three meshes $(5 \times 5,7 \times 7$, and $10 \times 10$ ) have been computed and compared with the analytical solution and ABAQUS shell element (S4 and S4R) solutions. The analytical solution of the natural frequency $\omega_{m n}$ for a simply supported rectangular plate is given [14]. The mode shapes of the first three modes are shown in Figure 5.

We use shell elements in ABAQUS's library (2004) for comparison with MP4 element for the plate bending results. In ABAQUS, triangular shell element with 3 nodes (STRI3) 


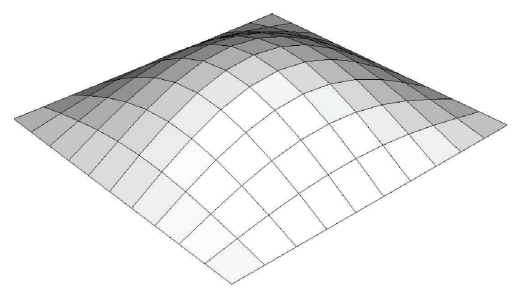

(a)

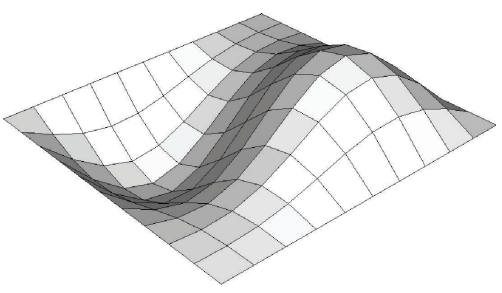

(b)

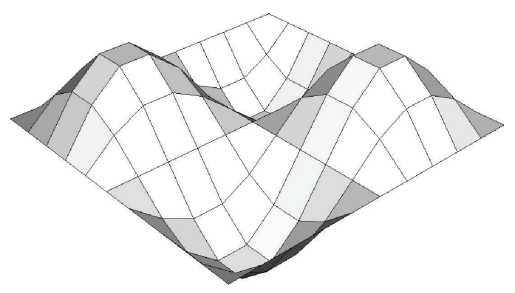

(c)

Figure 5: Example 1: mode shapes of the simply supported plate. (a) First mode shape; (b) second mode shape; (c) third mode shape (the fourth mode in actual analysis).

TABLE 1: Example 1: natural frequency of a simply supported plate with thickness $t=0.001 \mathrm{~m}$. (STRI3 is a Kirchhoff element with 3 nodes; S4 is an element with 4 nodes for thin or thick sections; S4R is an S4 element with reduced integration).

\begin{tabular}{|c|c|c|c|c|c|c|}
\hline$t / L=1 \%$ & Thin plate & Thick plate & STRI3 & S4 & S4R & MP4 (present) \\
\hline First mode & 484.33 & 484.19 & & & & \\
\hline $5 \times 5$ & & & 459.85 & 505.95 & 500.48 & 496.71 \\
\hline $7 \times 7$ & & & 485.26 & 494.88 & 492.23 & 490.12 \\
\hline $10 \times 10$ & & & 483.58 & 489.06 & 487.80 & 483.56 \\
\hline Second mode & 1210.80 & 1210.00 & & & & \\
\hline $5 \times 5$ & & & 1105.55 & 1434.10 & 1408.60 & 1347.10 \\
\hline $7 \times 7$ & & & 1200.35 & 1315.10 & 1303.70 & 1277.80 \\
\hline $10 \times 10$ & & & 1204.35 & 1258.90 & 1253.60 & 1241.80 \\
\hline Third mode & 1937.30 & 1935.10 & & & & \\
\hline $5 \times 5$ & & & 1625.90 & 2323.50 & 2206.80 & 2026.40 \\
\hline $7 \times 7$ & & & 1863.70 & 2118.60 & 2069.60 & 2000.90 \\
\hline $10 \times 10$ & & & 1915.50 & 2020.30 & 1998.60 & 1980.80 \\
\hline
\end{tabular}

TABLE 2: Example 1: natural frequency of a simply supported plate with thickness $t=0.005 \mathrm{~m}$.

\begin{tabular}{|c|c|c|c|c|c|c|}
\hline$t / L=5 \%$ & Thin plate & Thick plate & STRI3 & S4 & S4R & MP4 (present) \\
\hline First mode & 2421.70 & 2404.80 & & & & \\
\hline $5 \times 5$ & & & 2295.30 & 2482.90 & 2458.50 & 2426.10 \\
\hline $7 \times 7$ & & & 2421.50 & 2422.60 & 2410.90 & 2385.80 \\
\hline $10 \times 10$ & & & 2413.20 & 2388.50 & 2382.90 & 2397.70 \\
\hline Second mode & 6054.10 & 5950.20 & & & & \\
\hline $5 \times 5$ & & & 5509.10 & 6934.30 & 6826.40 & 6546.80 \\
\hline $7 \times 7$ & & & 5973.10 & 6371.20 & 6323.00 & 6210.80 \\
\hline $10 \times 10$ & & & 6044.21 & 6098.50 & 6076.40 & 6034.90 \\
\hline Third mode & 9686.60 & 9424.50 & & & & \\
\hline $5 \times 5$ & & & 8100.70 & 10967.00 & 10499.00 & 9716.10 \\
\hline $7 \times 7$ & & & 9256.20 & 10059.00 & 9860.60 & 9591.40 \\
\hline $10 \times 10$ & & & 9504.80 & 9604.30 & 9516.00 & 9427.90 \\
\hline
\end{tabular}

is recommended to be used for Kirchhoff element [19]. Quadrilateral elements S4 and S4R, with a hypothesis of linear interpolation in the transverse direction, are commonly used as either Kirchhoff or Mindlin elements depending on the plate thickness. The S4 element is a fully integrated, isoparametric shell element with 6 DOFs at all four corner nodes. The S4R element has the same behavior as S4 except that it uses the reduced integration method, which means it contains only one center Gauss point. We used three different meshes $(5 \times 5,7 \times 7$ and $10 \times 10)$ for the plate when MP4, S4, and S4R elements were used. Free mesh was used for STRI3 elements. The three corresponding mesh patterns with STRI3 contain 20 elements and 17 nodes, 52 elements and 37 nodes, and 108 elements and 69, nodes respectively.

Tables 1, 2, and 3 compare the natural frequencies of the present MP4 plate element with other available reference solutions in a simply supported plate with thicknesses of $t=0.001,0.005$, and $0.01 \mathrm{~m}$, respectively. We found that 
TABLE 3: Example 1: natural frequency of a simply supported plate with thickness $t=0.01 \mathrm{~m}$.

\begin{tabular}{|c|c|c|c|c|c|c|}
\hline$t / \mathrm{L}=10 \%$ & Thin plate & Thick plate & STRI3 & S4 & S4R & MP4 (present) \\
\hline First mode & 4843.3 & 4712.3 & & & & \\
\hline $5 \times 5$ & & & 4566.2 & 4738.6 & 4699.5 & 4625.7 \\
\hline $7 \times 7$ & & & 4813.1 & 4623.0 & 4603.4 & 4573.6 \\
\hline $10 \times 10$ & & & 4896.9 & 4559.7 & 4550.0 & 4561.0 \\
\hline Second mode & 12108.3 & 11335.5 & & & & \\
\hline $5 \times 5$ & & & 10902.0 & 12747.0 & 12595.0 & 12189.0 \\
\hline $7 \times 7$ & & & 11771.0 & 11796.0 & 11725.0 & 11637.0 \\
\hline $10 \times 10$ & & & 11808.5 & 11330.0 & 11296.0 & 11331.0 \\
\hline Third mode & 19373.2 & 17499.7 & & & & \\
\hline $5 \times 5$ & & & 16015.0 & 19263.0 & 18661.0 & 17443.0 \\
\hline $7 \times 7$ & & & 18131.0 & 17904.0 & 17635.0 & 17345.0 \\
\hline $10 \times 10$ & & & 18573.0 & 17205.0 & 17080.0 & 17228.6 \\
\hline
\end{tabular}

TABLE 4: Error comparison of natural frequency for $10 \times 10$ mesh.

\begin{tabular}{|c|c|c|c|c|c|c|c|}
\hline & & \multicolumn{2}{|c|}{ First mode } & \multicolumn{2}{|c|}{ Second mode } & \multicolumn{2}{|c|}{ Third mode } \\
\hline & & $\alpha$ & $\beta$ & $\alpha$ & $\beta$ & $\alpha$ & $\beta$ \\
\hline \multirow{4}{*}{$t / L=1 \%$} & $\mathrm{~S} 4$ & 0.0098 & 0.0101 & 0.0397 & 0.0404 & 0.0428 & 0.0440 \\
\hline & S4R & 0.0072 & 0.0075 & 0.0353 & 0.0360 & 0.0316 & 0.0328 \\
\hline & STRI3 & 0.0015 & 0.0013 & 0.0053 & 0.0047 & 0.0113 & 0.0101 \\
\hline & MP4 & 0.0016 & 0.0013 & 0.0256 & 0.0263 & 0.0224 & 0.0236 \\
\hline \multirow{4}{*}{$t / L=5 \%$} & $\mathrm{~S} 4$ & 0.0137 & 0.0068 & 0.0073 & 0.0249 & 0.0085 & 0.0191 \\
\hline & S4R & 0.0160 & 0.0091 & 0.0037 & 0.0212 & 0.0176 & 0.0097 \\
\hline & STRI3 & 0.0035 & 0.0035 & 0.0016 & 0.0158 & 0.0188 & 0.0085 \\
\hline & MP4 & 0.0100 & 0.0029 & 0.0032 & 0.0142 & 0.0265 & 0.0006 \\
\hline \multirow{4}{*}{$t / L=10 \%$} & S4 & 0.0586 & 0.0324 & 0.0643 & 0.0005 & 0.1119 & 0.0168 \\
\hline & S4R & 0.0606 & 0.0344 & 0.0671 & 0.0035 & 0.1184 & 0.0240 \\
\hline & STRI3 & 0.0111 & 0.0392 & 0.0248 & 0.0417 & 0.0413 & 0.0613 \\
\hline & MP4 & 0.0582 & 0.0321 & 0.0642 & 0.0004 & 0.1107 & 0.0155 \\
\hline
\end{tabular}

$\alpha$ : Error to thin theory, $\beta$ : Error to thick theory.

the MP4 element, when used in vibration analysis, is expected to perform better than the STRI3, S4, and S4R elements, and that the best results for the natural frequencies can be obtained with $10 \times 10$ mesh of the plate. Table 4 compares the error of these four elements relative to thin plate theory and to thick plate theory for $10 \times 10$ mesh. It can be seen that the STRI3 element only works for a thin plate, and the S4R element works better than S4 for the thin plate and it is the reverse for the thick plate. We may see that the reduced integration is more accurate for the thin plate element whereas the full integration for thick plate element. The developed MP4 element performs excellent in vibration analysis for both thin and thick plate, especially for moderately thick plate. Note that when the MP4 elements are used for moderately thick plate $(t / L=$ $5 \%)$, the first and third mode of the plate are closer to the thick plate theory solution $(\beta=0.0029$ and 0.0006$)$, whereas the second mode is closer to the thin plate theory solution $(\alpha=0.0032)$.

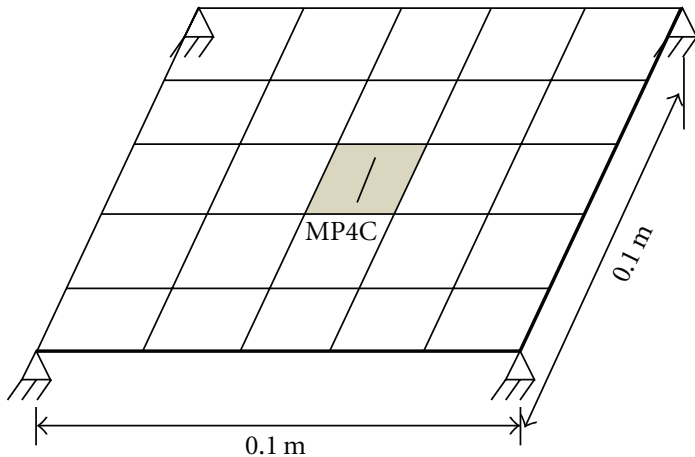

FIGURE 6: Example 2: mesh ( 5 by 5 ) of a simply supported plate with a center crack.

5.2. Example 2: Free Vibration of a Simply Supported Plate with a Through Crack. Figure 6 shows the plate with thickness of $0.001 \mathrm{~m}$ containing a crack in the middle of the plate. The crack is oriented parallel to the plate edges. A $5 \times 5$ mesh 
TABLE 5: Example 2: comparison of the influence of crack length on the ratios of the first four natural frequencies $\left(\Delta \omega_{i}=\omega_{i}^{c} / \omega_{i}\right)$ of the simply supported plate.

\begin{tabular}{|c|c|c|c|c|c|c|}
\hline Mode & $2 a / H$ & Stahl and Keer [17] & Solecki [18] & Guan-liang et al. [5] & Krawczuk et al. [8] & Present results \\
\hline \multirow{4}{*}{1 st } & 0.05 & 0.9970 & 0.9970 & 0.9975 & 0.9971 & 0.9991 \\
\hline & 0.10 & 0.9940 & 0.9940 & 0.9950 & 0.9942 & 0.9981 \\
\hline & 0.15 & 0.9860 & 0.9855 & 0.9885 & 0.9874 & 0.9933 \\
\hline & 0.20 & 0.9780 & 0.9775 & 0.9820 & 0.9806 & 0.9931 \\
\hline \multirow{4}{*}{ 2nd } & 0.05 & 0.9999 & 0.9999 & 0.9999 & 1.0000 & 1.0000 \\
\hline & 0.10 & 0.9996 & 0.9998 & 0.9999 & 1.0000 & 1.0000 \\
\hline & 0.15 & 0.9980 & 0.9982 & 0.9989 & 0.9980 & 0.9990 \\
\hline & 0.20 & 0.9980 & 0.9966 & 0.9980 & 0.9970 & 0.9982 \\
\hline \multirow{4}{*}{$3 \mathrm{rd}$} & 0.05 & 1.0000 & - & - & 1.0000 & 1.0000 \\
\hline & 0.10 & 1.0000 & - & - & 1.0000 & 1.0000 \\
\hline & 0.15 & 0.9999 & - & - & 0.9999 & 1.0000 \\
\hline & 0.20 & 0.9996 & - & 一 & 0.9998 & 0.9999 \\
\hline \multirow{4}{*}{4 th } & 0.05 & - & - & - & - & 1.0000 \\
\hline & 0.10 & - & - & - & - & 1.0000 \\
\hline & 0.15 & - & - & - & - & 1.0000 \\
\hline & 0.20 & - & - & - & - & 0.9999 \\
\hline
\end{tabular}

TABLE 6: Example 3: comparison of the ratios of the first four natural frequencies $\left(\Delta \omega_{i}=\omega_{i}^{c} / \omega_{i}\right)$ of a cantilever plate.

\begin{tabular}{lcccc}
\hline Mode & $\begin{array}{c}\text { Guan-liang } \\
\text { et al. [5] }\end{array}$ & Krawczuk [8] & $\begin{array}{c}\text { Guan-liang } \\
\text { et al. [5] } \\
\text { (Experiments) }\end{array}$ & $\begin{array}{c}\text { Present } \\
t / L=0.01\end{array}$ \\
\hline 1st & 0.9931 & 0.9891 & 0.9917 & 0.9940 \\
2nd & 0.9989 & 0.9985 & 0.9981 & 0.9992 \\
3rd & 0.9837 & 0.9826 & 0.9807 & 0.9910 \\
4th & - & - & - & 0.9923 \\
\hline
\end{tabular}

is used to model the plate, which includes $24 \mathrm{MP} 4$ elements and one MP4C element shaded in the figure. Changes in the first four natural frequencies of the plate due to crack are investigated. It should be noted that in [8] the influence of the crack location in the plate on the changes of natural frequencies was analyzed. In this example, the most critical crack for a simply supported plate is located at the position of local maxima of bending moments. The ratio of natural frequency of cracked plate to one of the plates without crack, $\Delta \omega_{i}=\omega_{i}^{c} / \omega_{i}$, is used as the testing index, and $\omega_{i}$ is the $i$ th natural frequency of noncracked plate, and $\omega_{i}^{c}$ is the $i$ th natural frequency of the cracked plate. The relative crack length $(2 a / H)$ considered ranges from $5 \%$ to $20 \%$ of the plate width.

Table 5 compares the ratios $\left(\Delta \omega_{i}\right)$ of natural frequencies of the developed MP4C element with other reference results for four modes. It is worth noting that the solutions in the references were obtained using the regular plate bending elements based on the Kirchhoff theory. The present results are in good agreement with the reference results. We observe that for the simply supported boundary condition, crack

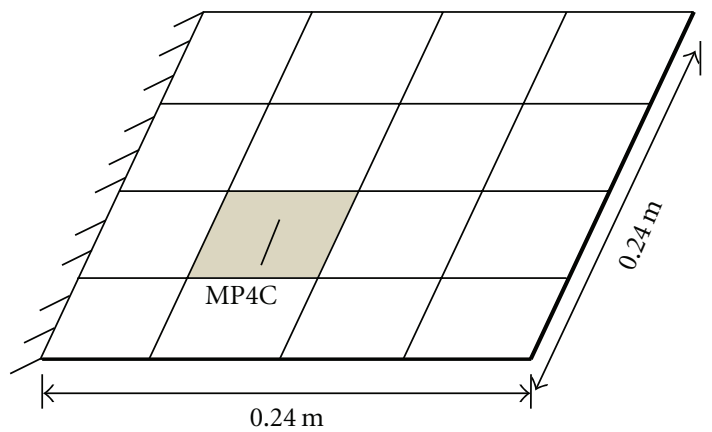

FIGURE 7: Example 3: mesh ( 4 by 4 ) of a cantilever plate with a crack.

has the most significant effects on the basic bending mode of plate. Note that the values of $\Delta \omega_{i}$ decrease as the crack length increases in each single mode, and for higher modes, the effect of crack is not as obvious as that on the lower modes. The present results are close to those obtained using the Kirchhoff plate models since the transverse shear term only has slight influence in the flexibility matrix of the Mindlin plate. However, we observe that the present results are generally larger than those of other references, which indicates the crack has less effect in the Mindlin plate model than in the Kirchhoff plate model. The reason is that the Mindlin plate element is more flexible than the Kirchhoff plate element because the Kirchhoff plate element neglects the transverse deformation on its cross-section which actually adds stiffness. Further investigation of this phenomenon is given in next example.

5.3. Example 3: Free Vibration of a Cantilever Plate with a Through Crack. This example focuses on the effect of crack 
TABLE 7: Example 3: the ratios of the first four natural frequencies $\left(\Delta \omega_{i}=\omega_{i}^{c} / \omega_{i}\right)$ of a cantilever plate with different thicknesses.

\begin{tabular}{lcccc}
\hline Mode & $t / L=0.001$ & $t / L=0.01$ & $t / L=0.05$ & $t / L=0.1$ \\
\hline 1st & 1.0000 & 0.9940 & 0.9840 & 0.9837 \\
2nd & 1.0000 & 0.9992 & 0.9943 & 0.9941 \\
3rd & 0.9999 & 0.9910 & 0.9735 & 0.9731 \\
4th & 0.9999 & 0.9923 & 0.9811 & 0.9803 \\
\hline
\end{tabular}

on the behavior of a cantilever square plate. The plate is made of aluminum with Young's modulus $E=6.9 \times 10^{10} \mathrm{~N} / \mathrm{m}^{2}$, Poisson ratio $v=0.33$, and density $\rho_{0}=2800 \mathrm{~kg} / \mathrm{m}^{3}$. The length of an edge of the square plate was $0.24 \mathrm{~m}$ and its thickness was $0.00275 \mathrm{~m}$. A through crack of length $2 a=$ $0.0035 \mathrm{~m}$ was intentionally introduced at $x=0.09 \mathrm{~m}, y=$ $0.09 \mathrm{~m}$, parallel to the clamped boundary (see Figure 7). In this case, a $4 \times 4$ mesh including 15 MP4 elements and one MP4C element is used.

Table 6 compares the ratios $\left(\Delta \omega_{i}\right)$ of the first four natural frequencies of the present results with those of available results in Guan-liang et al. [5] and Krawczuk et al. [8]. The present results show very good agreement with experimental results in Guan-liang et al. [5] and other available numerical results. Similar to the previous example, the larger values of $\Delta \omega_{i}$ are observed in the present model. Table 7 compares the ratios $\left(\Delta \omega_{i}\right)$ of the first four natural frequencies of the present results for four different thickness-to-length ratios: $t / L=0.1 \%, 1 \%, 5 \%$, and $10 \%$. We found that, with the same crack length, the thinnest plate $(t / L=0.1 \%)$ is subjected to the least influence on natural frequency. Significant changes in natural frequencies are observed in the case of the moderately thick plate $(t / L=5 \%)$ and the thick plate $(t / L=$ $10 \%)$ at the first and second modes, which shows that the crack effects on plates are modal dependent. The results in this example demonstrate that the plate thickness is a key factor when evaluating the effect of crack on plate bending behavior.

\section{Conclusions}

In this paper, we developed a new four-noded rectangular Mindlin plate finite element with a through crack. This element was used for vibration analyses based on plate bending. The performances of the MP4 and MP4C elements are compared with other available elements in the literature and also with ABAQUS shell elements (STRI3, S4, and S4R). Moreover, the developed elements are integrated into ABAQUS [1] as user elements.

From this study, we may conclude the following.

(i) The formulation of the MP4 element, which is free of shear locking, is simple and straightforward. As evident through comparison with the thin and thick plate theories, the proposed MP4 element has shown better accuracy for natural frequencies than conventional shell elements available in ABAQUS. In particular, the proposed element yields better accuracy for moderately thick plates, which are more common types of plates in engineering practice.

(ii) The local flexibility method is used to develop the cracked MP4C element based on the noncracked MP4 element. Mixed-mode SIFs have been independently introduced to fully represent the effect of crack on the flexibility matrix of the Mindlin plate element. We observe that dynamical behaviors of plate structures are influenced by the crack length, crack location, boundary conditions, and plate thickness.

(iii) The present energy-based approach efficiently addresses the global vibration behavior of plate structures considering strain energy perturbation induced by crack and thus makes computational efficiency possible without the need for a highly dense mesh around crack tips. Moreover, the integration of MP4 and MP4C elements into FEM packages, such as ABAQUS, provides an efficient tool to model the static, dynamic, and stability behaviors of plate-like structures with multiple cracks.

\section{Appendix}

A.1.

The transformation matrix $[T]$ is

$$
[T]=\left[\begin{array}{ccccccccc}
0 & \frac{2}{H} & 0 & 0 & 0 & 0 & 0 & -\frac{2}{L} & -1 \\
1 & 1 & 0 & 0 & 0 & 0 & 0 & 0 & 0 \\
0 & 0 & 0 & 0 & 0 & 0 & -1 & 1 & 0 \\
0 & -\frac{2}{H} & 0 & \frac{2}{L} & 0 & 0 & 0 & 0 & 1 \\
-1 & 1 & 0 & 0 & 0 & 0 & 0 & 0 & 0 \\
0 & 0 & -1 & -1 & 0 & 0 & 0 & 0 & 0 \\
0 & 0 & 0 & -\frac{2}{L} & 0 & \frac{2}{H} & 0 & 0 & -1 \\
0 & 0 & 0 & 0 & -1 & -1 & 0 & 0 & 0 \\
0 & 0 & 1 & -1 & 0 & 0 & 0 & 0 & 0 \\
0 & 0 & 0 & 0 & 0 & -\frac{2}{H} & 0 & \frac{2}{L} & 1 \\
0 & 0 & 0 & 0 & 1 & -1 & 0 & 0 & 0 \\
0 & 0 & 0 & 0 & 0 & 0 & 1 & 1 & 0
\end{array}\right]
$$

\section{A.2.}

The flexibility matrix of the analyzed element without the crack $\left[C_{f}\right]^{0}$ is derived in this paper 


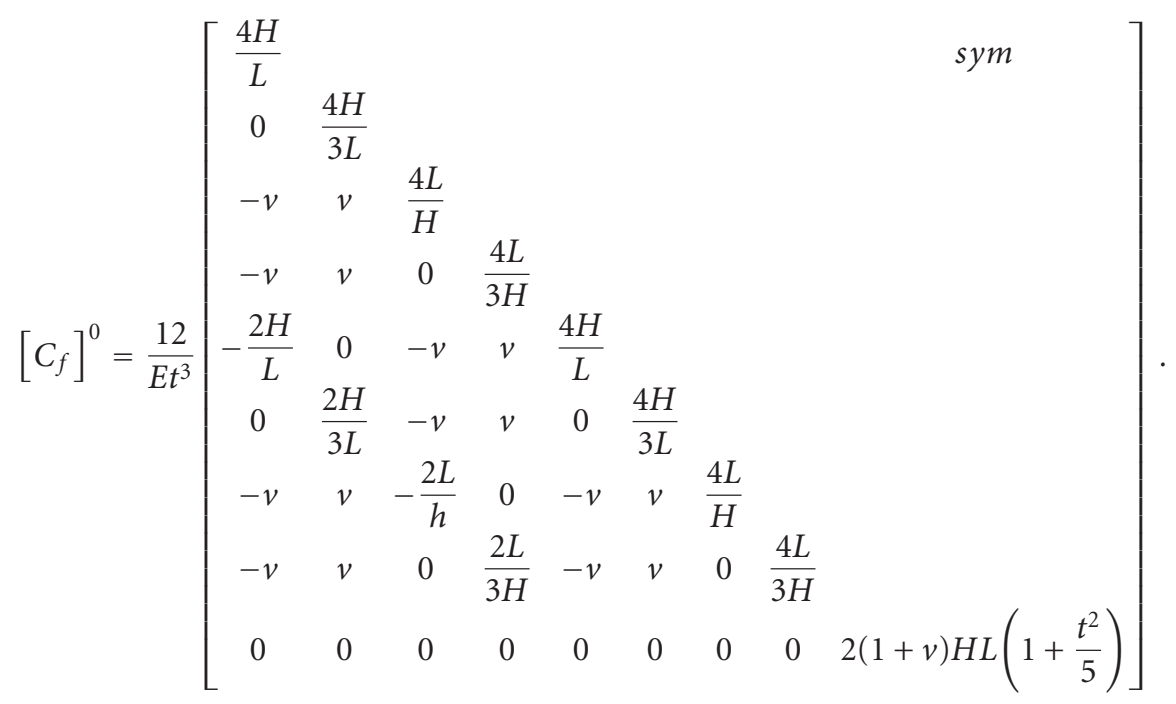

A.3.

The flexibility matrix of the analyzed element with crack $\left[C_{f}\right]^{1}$ is

$$
\left[C_{f}\right]^{1}=\frac{6 \pi}{E t^{3}}\left[\begin{array}{ccccccccc}
0 & & & & & & & & s y m \\
0 & 0 & & & & & & & \\
0 & 0 & c_{33} & & & & & & \\
0 & 0 & 0 & 0 & & & & & \\
0 & 0 & 0 & 0 & 0 & & & & \\
0 & 0 & 0 & 0 & 0 & 0 & & & \\
0 & 0 & c_{37} & 0 & 0 & 0 & c_{77} & & \\
0 & 0 & 0 & 0 & 0 & 0 & 0 & 0 & \\
0 & 0 & 0 & 0 & 0 & 0 & 0 & 0 & c_{99}
\end{array}\right] .
$$

\section{Nomenclature}

a: Half crack length

A: $\quad$ Area of the plate element

$A^{1}$ : $\quad$ Area of the crack

$[B]$ : Derivative of the shape function matrix

$\left[B_{b}\right]$ : Bending terms in $[B]$

$\left[B_{s}\right]$ : Transverse shear terms in $[B]$

$c_{i, j}^{1}$ : Terms in the flexibility matrix due to the crack

$[C]$ : Damping matrix in governing equation for structural dynamics

$\left[C_{f}\right]$ : Flexibility matrix of the cracked Mindlin plate element

$\left[C_{f}\right]^{0}$ : Flexibility matrix of the Mindlin plate element without crack

$\left[C_{f}\right]^{1}$ : Flexibility matrix due to the crack

$\{d\}$ : Nodal displacement vector in governing equation for structural dynamics

$[D]$ : Flexural rigidity matrix è: $\quad$ Stress distribution due to unit force in plate

$E$ : $\quad$ Elastic modulus of the plate

$F_{1}-F_{9}$ : Independent nodal forces on the Mindlin plate element

$H: \quad$ Width of the plate element

$K_{i}: \quad$ Stress intensity factors, $i=I, I I, I I I$

$\{\kappa\}: \quad$ Curvature and strain column vector

$[K]: \quad$ Stiffness matrix of the Mindlin plate element

$\left[K_{c}\right]: \quad$ Stiffness matrix of the cracked Mindlin plate element

(A.3) L: $\quad$ Length of the plate element

$\{M c\}$ : Moment and shear force column vector

$N_{i}(\xi, \eta)$ : Shape function at node $i$ for the four-node Mindlin plate element

$\left\{R^{\text {ext }}\right\}$ : External force vector in governing equation for structural dynamics

$S_{1}-S_{12}$ : Nodal forces on the Mindlin plate element

$t$ : Thickness of the plate element

[T]: Transformation matrix

$u$ : $\quad$ Displacement of the plate midsurface in positive $x$-direction

$U: \quad$ Strain energy in the element

$U^{1}: \quad$ Additional strain energy due to the crack

$v$ : Displacement of the plate midsurface in positive $y$-direction

$w$ : $\quad$ Displacement of the plate midsurface in positive $z$-direction

$Y(g)$ : Correction function for the infinite dimension of element to finite dimension

$\varepsilon_{x}$ : $\quad$ Normal strain of the plate in $x$-direction

$\varepsilon_{y}: \quad \quad \quad \quad \quad$ ormal strain of the plate in $y$-direction

$\gamma_{x y}: \quad$ Shear strain in $x y$ plane of the plate

$\gamma_{y z}$ : Transverse shear strain in $y z$ plane of the plate

$\gamma_{z x}$ : Transverse shear strain in $z x$ plane of the plate

$\rho_{0}$ : $\quad$ Mass density of the plate

$v$ : $\quad$ Poisson ratio of the plate 
$\Delta \omega_{i}: \quad$ Ratio of frequencies of cracked plate to one of the plate without crack, $\Delta \omega_{i}=\omega_{i}^{c} / \omega_{i}$

$\omega_{i}$ : $\quad i$ th natural frequency of noncracked plate

$\omega_{i}^{c}: \quad i$ th natural frequency of the cracked plate

$\sigma_{x}(0, y)$ : Normal stress acting on the crack face

$\tau_{x y}(0, y)$ : Shear stress acting on the crack face

$\psi_{x}$ : $\quad$ Rotation of the plate midsurface pointing to negative $y$-direction

$\psi_{y}: \quad$ Rotation of the plate midsurface pointing to positive $x$-direction

$[\Phi]$ : $\quad$ Inverse matrix of flexural rigidity.

\section{Acknowledgments}

This work has been carried out at the University of Connecticut as part of a research project on bridge monitoring directed by the middle author. The support of the Connecticut Department of Transportation and the U.S. Department of Transportation, Federal Highway Administration is gratefully acknowledged. The work was done with further support from the Connecticut Transportation Institute at the University of Connecticut. This work is an outcome of a course taught by the last author. The start-up support provided to him by the University of Connecticut is gratefully acknowledged.

\section{References}

[1] J. T. DeWolf, J. K. Olund, C. Liu, and A. J. Cardini, "The long term structural health monitoring of bridges in the state of Connecticut," in Proceedings of the 3rd European Workshop on Structural Health Monitoring (SHM '06), Granada, Spain, July 2006.

[2] C. Liu and J. T. DeWolf, "Effect of temperature on modal variability of a curved concrete bridge under ambient loads," Journal of Structural Engineering, vol. 133, no. 12, pp. 17421751, 2007.

[3] W. J. Thomson, "Vibration of slender bars with discontinuities in stiffness," Journal of Applied Mechanics, vol. 17, pp. 203-207, 1943.

[4] P. G. Kirmsher, "The effect of discontinuities on the natural frequency of beams," Proceeding of American Society of Testing and Materials, vol. 44, pp. 897-904, 1944.

[5] Q. Guan-Liang, S. N. Gu, and J. S. Jiang, "A finite element model of cracked plates and application to vibration problems," Computers and Structures, vol. 39, no. 5, pp. 483-487, 1991.

[6] M. Krawczuk, "A rectangular plate finite element with an open crack," Computers and Structures, vol. 46, no. 3, pp. 487-493, 1993.

[7] M. Krawczuk, "Rectangular shell finite element with an open crack," Finite Elements in Analysis and Design, vol. 15, no. 3, pp. 233-253, 1994.

[8] M. Krawczuk, A. Zak, and W. Ostachowicz, "Finite element model of plate with elasto-plastic through crack," Computers and Structures, vol. 79, no. 5, pp. 519-532, 2001.

[9] R. D. Mindlin, "Influence of rotatory inertia and shear on flexural motions of isotropic, elastic plates," ASME Journal of Applied Mechanics, vol. 18, pp. 31-38, 1951.

[10] J. H. Kim and G. H. Paulino, "Finite element evaluation of mixed mode stress intensity factors in functionally graded materials," International Journal for Numerical Methods in Engineering, vol. 53, no. 8, pp. 1903-1935, 2002.

[11] ABAQUS/Analysis User's Manual, Version 6.5. Hibbit, Karlsson and Sorenson, 2004.

[12] R. D. Cook, D. S. Malkus, M. E. Plesha, and R. J. Witt, Concepts and Applications of Finite Element Analysis, John Wiley, New York, NY, USA, 4th edition, 2001.

[13] J. S. Przemieniecki, Theory of Matrix Structural Analysis, McGraw-Hill, New York, NY, USA, 1968.

[14] A. C. Ugural, Stresses in Plate and Shells, McGrawHill, New York, NY, USA, 1999.

[15] G. C. Sih and H. Liebowitz, Mathematical Theory of Brittle Fracture, Math Fundmamental, New York, NY,USA, 1968.

[16] G. Yagawa and T. Nishioka, "Finite element analysis of the stress intensity factors for plane extension and plate bending problem," International Journal for Numerical Methods in Engineering, vol. 14, no. 5, pp. 727-740, 1979.

[17] B. Stahl and L. M. Keer, "Vibration and stability of cracked rectangular plates," International Journal of Solids and Structures, vol. 8, no. 1, pp. 69-91, 1972.

[18] R. Solecki, "Bending vibration of a simply supported rectangular plate with a crack parallel to one edge," Engineering Fracture Mechanics, vol. 18, no. 6, pp. 1111-1118, 1983.

[19] G. Bezine, "On a method of comparison for plate elements in finite element engineering software programs," Mechanics Research Communications, vol. 29, no. 1, pp. 35-43, 2002. 

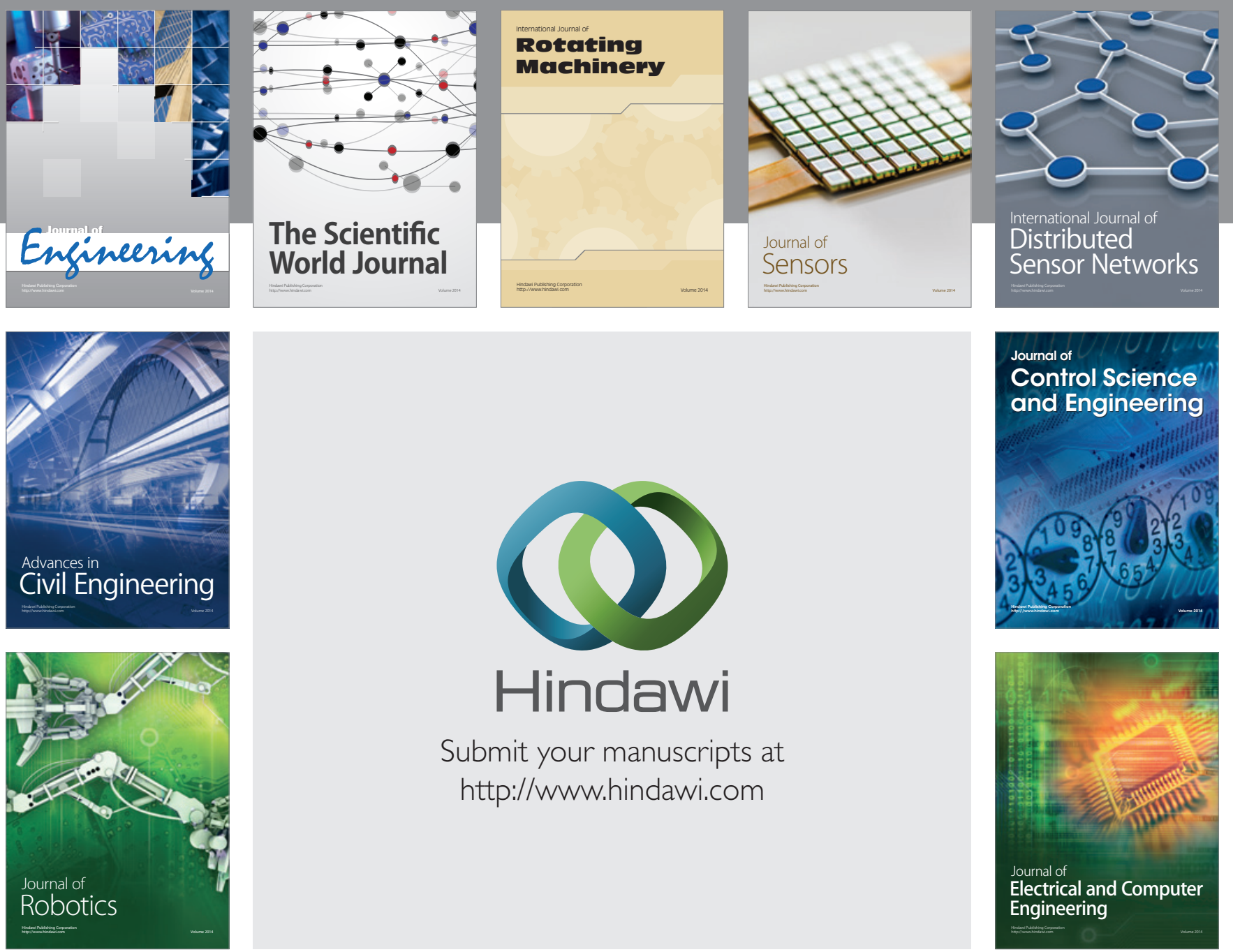

Submit your manuscripts at

http://www.hindawi.com
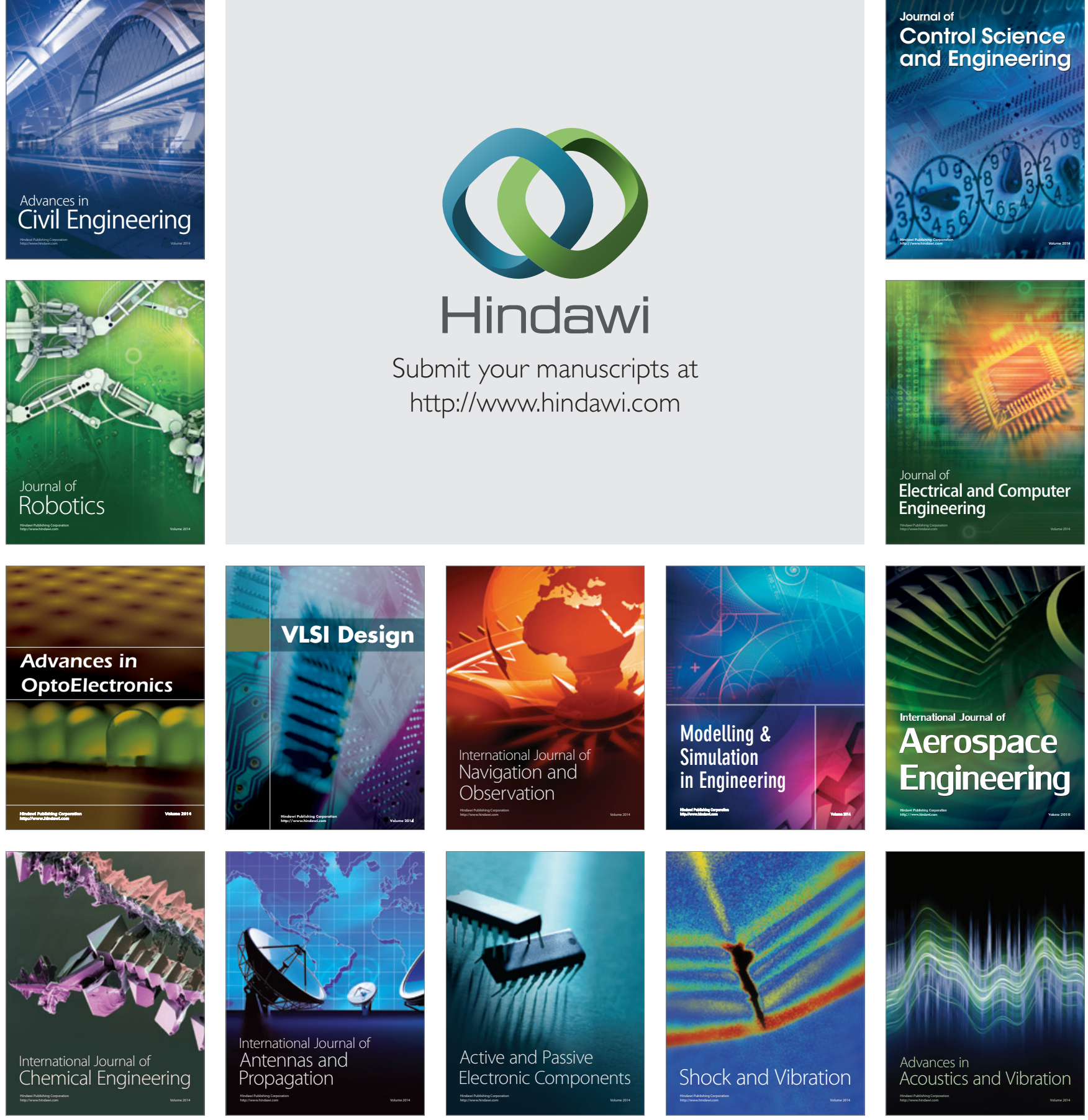